\title{
ARTICLE OPEN \\ Observational study: microgravity testing of a phase-change reference on the International Space Station
}

\author{
T Shane Topham ${ }^{1}$, Gail E Bingham ${ }^{1}$, Harri Latvakoski ${ }^{1}$, Igor Podolski ${ }^{2}$, Vladimir S Sychev ${ }^{2}$ and Andre Burdakin ${ }^{3}$
}

BACKGROUND: Orbital sensors to monitor global climate change during the next decade require low-drift rates for onboard thermometry, which is currently unattainable without on-orbit recalibration. Phase-change materials (PCMs), such as those that make up the ITS-90 standard, are seen as the most reliable references on the ground and could be good candidates for orbital recalibration. Space Dynamics Lab (SDL) has been developing miniaturized phase-change references capable of deployment on an orbital blackbody for nearly a decade.

AIMS: Improvement of orbital temperature measurements for long duration earth observing and remote sensing.

METHODS: To determine whether and how microgravity will affect the phase transitions, SDL conducted experiments with ITS-90 standard material (gallium, Ga) on the International Space Station (ISS) and compared the phase-change temperature with earth-based measurements. The miniature on-orbit thermal reference (MOTR) experiment launched to the ISS in November 2013 on Soyuz TMA-11M with the Expedition $38 \mathrm{crew}$ and returned to Kazakhstan in March 2014 on the Soyuz TMA-10 spacecraft. RESULTS: MOTR tested melts and freezes of Ga using repeated 6-h cycles. Melt cycles obtained on the ground before and after launch were compared with those obtained on the ISS.

CONCLUSIONS: To within a few mK uncertainty, no significant difference between the melt temperature of Ga at $1 \mathrm{~g}$ and in microgravity was observed.

npj Microgravity (2015) 1, 15009; doi:10.1038/npjmgrav.2015.9; published online 20 August 2015

\section{INTRODUCTION}

The Committee for Earth Science and Applications from Space has articulated a scientific need for orbital temperature knowledge to support infrared radiance measurements with $0.1-\mathrm{K}$ uncertainty ${ }^{1}$ over a period of at least 10 years. As temperature uncertainty is only one of the contributors to the desired $0.1-\mathrm{K}$ uncertainty, the goal for on-orbit temperature knowledge must be smaller (in the range of $0.01 \mathrm{~K}$ ) to leave margin for other uncertainties in the calibration chain. Onboard references utilizing phase transitions have been identified as the most likely means for realizing International System of Units (SI) traceability for temperature measurements in orbit.

The ITS-90 (refs. 2,3) identifies several phase-change materials (PCMs) with reliable fixed points that can be reproduced and used as references with submilli-kelvin absolute uncertainties for ground-based calibrations. Three PCMs with fixed points in the range required to calibrate Earth-observing sensors are gallium (Ga), water, and mercury with melt points of $302.9146,273.16$, and $234.3156 \mathrm{~K}$, respectively. ${ }^{3}$

However, the ITS-90 description of procedures and apparatuses does not translate easily into a design for an automated orbital implementation. ITS-90 describes fixed-point cells that use fragile materials such as plastic or glass to contain PCMs and require sensors to be placed in reentrant wells within relatively large volumes of $250 \mathrm{ml}$ or more of the PCM. The described procedures do not apply directly to in situ sensor calibrations such as those that will be required on an orbital blackbody.

In early 2006, the suitability of Ga and its various binary eutectics were investigated at the All Russian Institute for Opto-Physical
Measurements (VNIIOFI) in Moscow, Russia under a subcontract with Space Dynamics Laboratory (SDL) in Logan, UT, USA., ${ }^{4,5}$ The purpose of these studies was to significantly reduce the size and volume of qualified containers and identify PCMs within the range of $273-303 \mathrm{~K}$ that could be used as calibration standards aboard Earth-observing satellites. This study was successful in identifying and characterizing several suitable eutectics to fill the gap in the ITS-90 temperature scale between the triple point of water and the melting point of $\mathrm{Ga}$. SDL has continued research to further reduce the size and minimize volume of PCMs for an orbital thermal reference cell. The VNIIOFI developed cell ${ }^{6}$ held $22 \mathrm{ml}$ of $\mathrm{Ga}$, whereas the cell flow in the miniature on-orbit thermal reference (MOTR) experiment held about $1 \mathrm{ml}$.

Independent orbital studies that included phase changes of various materials have shown that crystallization structures in material solidified in the absence of gravity can differ from solids formed at $1 \mathrm{~g} .{ }^{7,8}$ Because the basis of these prior investigations was not temperature calibration or repeatability of fixed points, no accurate temperature data were recorded. The temperature of phase transitions is unlikely to be affected by gravity, but there was a lack of evidence to support that hypothesis.

SDL and the Institute for Biomedical Problems (IBMP) in Moscow, Russia have worked for several years to flight test PCM experiments in the Russian section of the ISS with the objective of verifying the utility of phase-change references in microgravity. IBMP and SDL had already partnered for over two decades conducting plant growth research on the Mir Space Station and ISS, and easily expanded their partnership for the MOTR experiments.

\footnotetext{
${ }^{1}$ Space Dynamics Laboratory (SDL), Utah State University Research Foundation, North Logan, UT, USA; ${ }^{2}$ Institute of Biomedical Problems of the Russian Academy of Sciences (SSCRF-IBMP RAS), Moscow, Russia and ${ }^{3}$ Russian Scientific Research Institute for Optical and Physical Measurements (VNIIOFI), Moscow, Russia.

Correspondence: TS Topham (shane.topham@sdl.usu.edu)

Received 29 April 2015; revised 9 June 2015; accepted 9 July 2015
} 


\section{MATERIALS AND METHODS}

In 2009, a phase-change cell and test hardware was designed and built at SDL for use on the ISS. This hardware was delivered to Moscow in December 2010, and launched to the ISS in November 2013. Data were collected onboard the ISS during January 2014, after which the unit returned to Earth in March 2014 and was delivered to SDL for post-flight testing.

\section{Hardware description}

The ISS Ga-MOTR experiment package consisted of two modules: experiment module (EM) and power module. The EM contains the PCM melt block and all of the electronics required to perform melts and freezes and is designed around a SensorCore-embedded computer manufactured by Tern Inc. (Davis, CA, USA). Experiment data, including temperatures, are stored on a nonvolatile memory card. The EM is $14.3 \times 8.0 \times 6.4 \mathrm{~cm}$ and the power module is $14.7 \times 7.6 \times 2.9 \mathrm{~cm}$.

The SensorCore computer is fully programmable and supports input through multiple 24-bit Analog to Digital (A/D) converters. Temperature and other system data are sampled by the $A / D$ converters approximately once in every $0.5 \mathrm{~s}$. Three to four consecutive samples are averaged by the software during each write interval and the averaged measurements are written to a nonvolatile compact flash memory card once in every $2.2 \mathrm{~s}$.

The EM houses the PCM cell, instrumented with temperature sensors and thermal-control elements. This PCM cell, which contained $1 \mathrm{ml}$ of Ga, had an expandable stainless steel bellows that allowed for expansion of the $\mathrm{Ga}$ during phase transitions without causing melt-point inaccuracies due to pressure changes. All wetted material is stainless steel to avoid PCM contamination. Laser welding produces a hermetic container with no moving seals, minimizing the chance of a container breach. This cell benefits from having a reentrant well where the temperature sensor is completely surrounded by the PCM. The cell can be heated and cooled approximately $\pm 30^{\circ} \mathrm{C}$ from cabin temperature with a 15 -Watt Peltier thermoelectric cooler (TEC). The temperature sensor in the phase-change cell well is a precision low-drift Thermometric SP60 thermistor.

The complete measurement stack, including the lower TEC used to transfer heat to the air-cooled heat sink, is shown in Figure 1. The PCM cell is housed in a slightly larger diameter container supporting the printed circuit heater used to bring it slowly through the melt temperature. Both the heater container and the PCM cell are mounted to the upper TEC. The cell container occupies approximately one cubic inch inside the EM housing. On the other side of the upper TEC is a blackbody simulator block which is mounted to a heat sink through a second TEC. Temperature sensors are located on the block and heat sink. The cell unit and the TEC stack are contained in a larger, insulated container mounted on the TEC heat sink to minimize the effects of ambient temperature swings. This three-container system meets the triple containment safety requirement applied to hazardous materials in ISS hardware.

An array of tests were performed on the flight unit before delivery as per Russian protocols to verify safety, compatibility, and survivability requirements for space travel.

\section{Experiment procedure}

The EM was programmed to perform repeated melt cycles automatically after the unit was turned on. A melt cycle started with both TECs in maximum cool mode to freeze the Ga PCM. As Ga, which melts at $29.7646^{\circ} \mathrm{C}$, can supercool significantly depending on preconditioning, the routine was programmed to cool the cell to $-10^{\circ} \mathrm{C}$ unless a freeze was detected. During a freeze, the cell temperature will stabilize for a period as the latent heat of freezing is released, and then continue cooling. When a thermal transient of this nature was detected, or after a time out period had been reached while the cell was being held at $-10^{\circ} \mathrm{C}$, the unit was programmed to prepare for a melt by bringing the cell to $0.1{ }^{\circ} \mathrm{C}$ below the expected $\mathrm{Ga}$ melt temperature and allowing the system to stabilize thermally. After all monitored temperatures had stabilized, the melt heater shown in Figure 1 was energized and controlled to maintain a constant temperature of $30^{\circ} \mathrm{C}$. This added the gentle heating necessary to bring the cell slowly through the melt temperature.

The computer was programmed to allow a total of $6 \mathrm{~h}$ for each freezemelt cycle with $\sim 1 \mathrm{~h}$ of freeze and preparation activity, allowing for a 5-h melt period. At the end of each 6-h period, the process was repeated. For the first cycle after the unit was started, the unit generally cooled well below the Ga melt point before the Ga froze, but in the following cycles $\mathrm{Ga}$ freezes were consistently detected above $25^{\circ} \mathrm{C}$. Minimal supercooling in

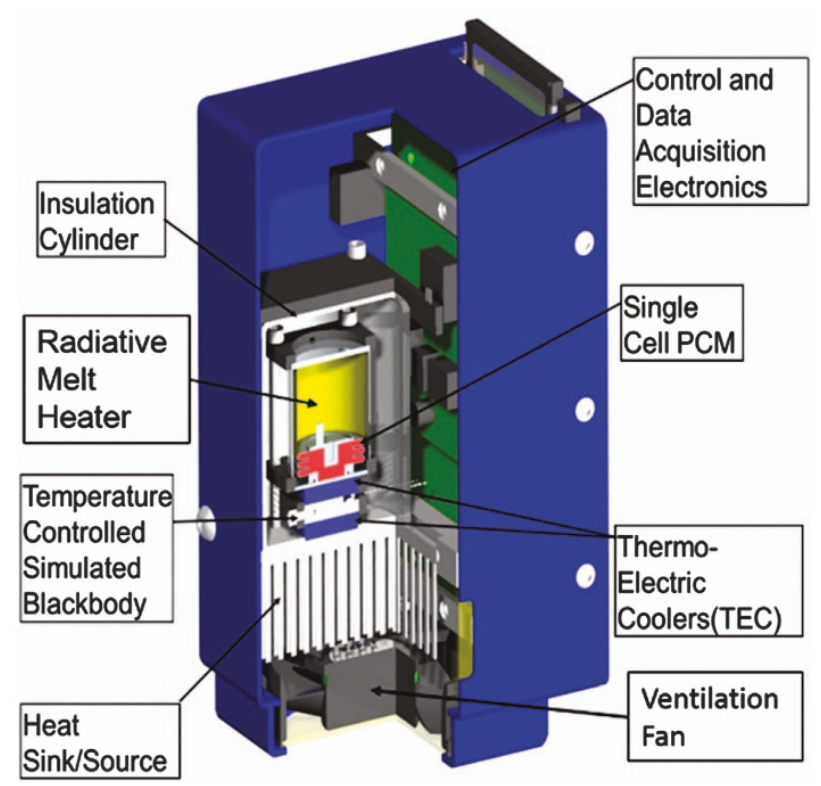

Figure 1. Cutaway illustration of the complete measurement stack inside the gallium $(\mathrm{Ga})$-the miniature on-orbit thermal reference (MOTR) Experiment Module $(14.3 \times 8.0 \times 6.4 \mathrm{~cm})$. PCM, phase-change material.

these following cycles is due to the lack of significant PCM temperature excursions above the Ga melt temperature, which tend to cause greater supercooling behaviors.

\section{Sensor calibration}

SDL maintains National Institute of Standards and Technology (NIST)traceable temperature calibration standards and these were used in the post-flight calibration and testing of the Ga-MOTR instrument. The calibration used a Fluke 7341 high-precision temperature-controlled bath (Fluke, Everett, WA, USA), with $\pm 2.5 \mathrm{mK}$ stability and a Hart Scientific 5615 Secondary Reference platinum resistance thermometer (PRT) (Hart Scientific, American Fork, UT, USA), with a current NIST traceable calibration report with $\pm 5 \mathrm{mK}$ absolute uncertainty. The PRT was read by a calibrated Fluke 1560 BlackStack (Fluke) with 8-channel PRT Scanner module 2562 with an uncertainty of $\pm 10 \mathrm{mK}$. Calibration in the bath is performed by placing the thermistor to be calibrated in the bath near the reference PRT, having the bath go through several temperatures and hold for an hour at each, and using the average thermistor resistance and reference PRT temperature from each bath temperature setting to calculate calibration coefficients for the thermistor.

After return to SDL, the EM was carefully disassembled and the melt cell, with its embedded thermistor, was placed in a thin plastic bag and inserted into a stirred alcohol bath adjacent to the calibrated secondary reference PRT probe. The cell thermistor was read using the flight electronics.

For the post-flight calibration, the bath was programmed to dwell at six temperatures between 29.6 and $30^{\circ} \mathrm{C}$ to collect comparison temperature data. Prior to these measurements, the Ga was heated above its melt temperature enough that it would supercool as a liquid to below $29.6^{\circ} \mathrm{C}$ to ensure that no phase transitions occurred during the calibration data collection. Calibration coefficients were calculated from this data and are applied to all the flight and ground data. After calibration, the PRT and the cell thermistor temperature readings were within $1 \mathrm{mK}$, but the absolute accuracy of the thermistor as traceable through the Blackstack and PRT probe combination is $\sim 11 \mathrm{mK}$. Melt profiles that were collected in the bath by applying increasing rapid melts are shown in Figure 2. After lowering the bath temperature to freeze the $\mathrm{Ga}$, the bath temperature was reset to five separate temperatures between 30.25 and $31.25^{\circ} \mathrm{C}$, resulting in melt profiles lasting between 2.5 and $9 \mathrm{~h}$. All melt profiles average at mid-melt to $29.772{ }^{\circ} \mathrm{C} \pm 1 \mathrm{mK}$

The melt point of the $\mathrm{Ga}$ in the Ga-MOTR cell in the bath was determined by partially melting the $\mathrm{Ga}$ and adjusting the bath temperature in mid-melt until there was no difference between the bath and cell temperature. This is the bi-phase thermal equilibrium point. Using this process, the average reading during melting of the PCM (the lower curve in 


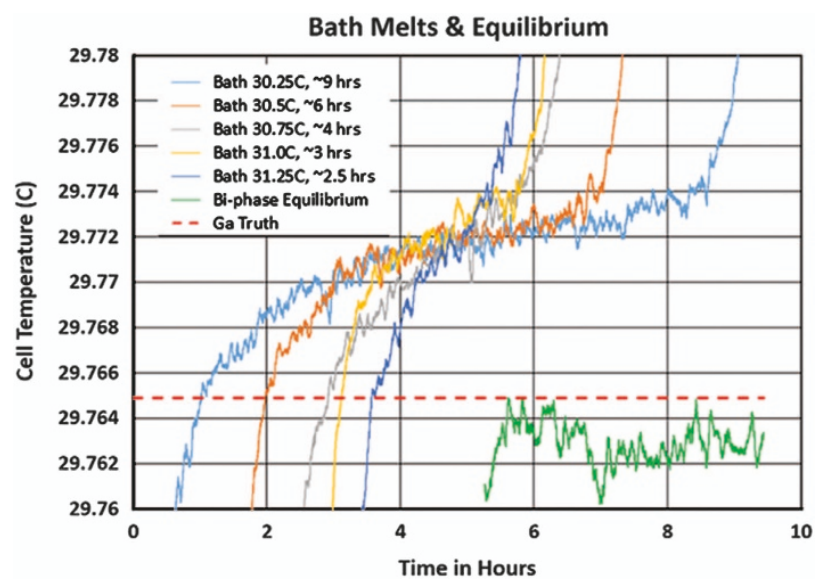

Figure 2. Melt profiles collected in the calibration bath using the flight electronics, but with the temperature change driven by the surrounding bath fluid. The uncertainty in absolute temperature is $11 \mathrm{mK}$. The starting times have been altered to show the grouping of the melt center temperature readings.

Table 1. Measurement timing summary for the Ga-MOTR unit

\begin{tabular}{lcl}
\hline Measurement/event & Date & Location \\
\hline SDL predelivery runs 1 and 2 & 1-24 November & SDL \\
& 2010 & \\
Moscow delivery & 3-5 December 2010 & IBMP \\
Moscow prelaunch runs 1 and 2 & 20-24 September & Moscow \\
& 2013 & \\
Launch on Soyuz (no data) & 6 November 2013 & Soyuz \\
ISS flight & 8-13 January 2014 & ISS \\
Return on Soyuz (no data) & 10 March 2014 & Soyuz \\
Moscow postflight run 1 and 2 & 18-29 April 2014 & IBMP \\
Hardware to Utah (no data) & 28 June 2014 & SDL \\
SDL postflight & 30 June 2014 to & SDL \\
& 5 July 2014 & \\
Temperature trend runs 1 and 2 & 17-18 July 2014 & SDL \\
Post recalibration & 1-6 October 2014 & SDL \\
\hline
\end{tabular}

Ga, gallium; IBMP, Institute for Biomedical Problems; ISS, International Space Station; MOTR, miniature on-orbit thermal reference; SDL, Space Dynamics Lab.

Figure 2), was measured to be about $1.5 \mathrm{mK}$, less than the ITS-90 Ga melt temperature of $29.7646{ }^{\circ} \mathrm{C}$. This is well within the absolute uncertainty of the cell thermistor as calibrated by the PRT and blackstack combination.

The melt curves obtained using this cell, as well as other small phasechange cells designs used, generally do not flatten out at the Ga melt point. A phase-change cell is nevertheless useful as an on-orbit temperature reference if the melt curves obtained are highly repeatable in temperature over time and from the ground to on-orbit. This repeatability is demonstrated in the Ga melt curves. The accuracy in using the cell as a temperature reference is limited by the ability to absolutely calibrate the cell thermistor on the ground. This could be improved over the $11 \mathrm{mK}$ obtained by calibrating with more accurate equipment.

\section{RESULTS}

Melt curves were collected with the test unit several times on the ground both before and after flight, as well as on the ISS. Program timing is shown in Table 1. Owing to programmatic issues not associated with the hardware, launch of the experiment to ISS was delayed by nearly 3 years after delivery to Moscow in 2010. The unit was in storage at IBMP until late 2013. Just before transportation to the launch site in preparation for launch, the unit was removed from storage and a preflight measurement

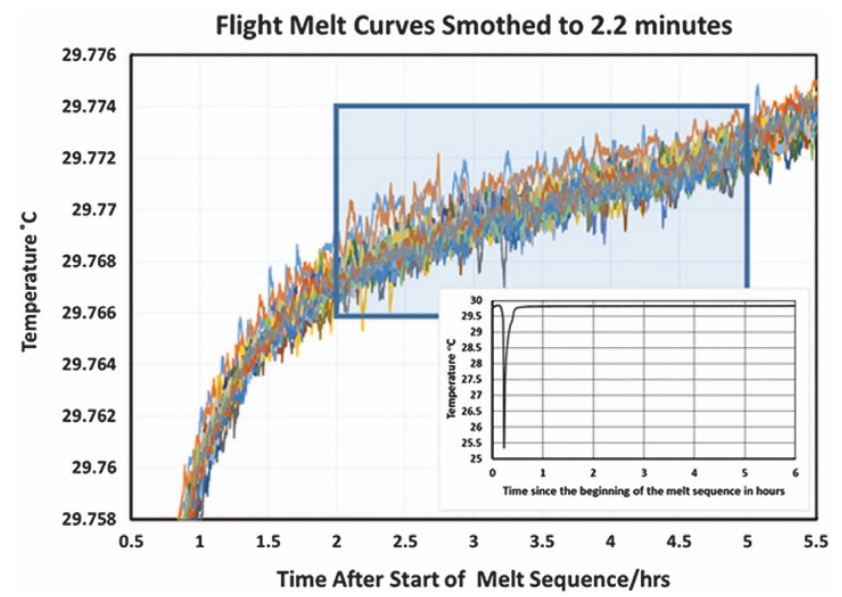

Figure 3. The melting portion of the 19 curves obtained by the flight unit on the ISS in January 2014. The inset shows the temperature over a single freeze and melt cycle.

sequence was conducted to ensure fitness for launch. The unit was installed on the ISS on 6 January 2014, and the measurement sequence was initiated on 8 January 2014.

After the initial startup sequences and conditioning, the unit successfully collected 19 melt curves. For each curve, the thermistor resistance was converted to temperature using the temperature calibration obtained post flight as described previously. For all data, the thermistor resistance was corrected for electronics drift using real-time measurements of a precision lowdrift resistor read with the same instrumentation and at the same rate as the thermistors. This is a standard correction for electronics drift where the measured resistance of the resistor is compared with its initial measured value and a correction based on this difference is applied to the thermistor data. During post-flight testing, the phase cell heater current was found to affect the thermistor reading at the $10 \mathrm{mK}$ level without affecting the precision resistor reading. This effect was measured during the bath testing, when the heater was physically detached from the phase-change cell but electrically connected, by simply changing the current on the heater and observing the change in measured thermistor temperature, while the cell was at a constant temperature in the bath. A correction based on the heater current and its measured effect on the temperature reading has been applied to all thermistor data.

The inset plot in Figure 3 shows the temperature over a single representative freeze-melt cycle. The timing of events was consistent to within minutes from cycle to cycle throughout the ground and flight experiments. During the first hour of each cycle, the Ga was refrozen and then prepared for a slow melt. From hours 1 to 5.5, the Ga was slowly melted. Figure 3 shows the melting portion of all 19 ISS flight cycles. The data were smoothed using a running average with a window of 59 samples, or $\sim 2$ min.

To facilitate comparison of melt curves collected at various times during the 4-year program (Table 1), melt temperature data between 2 and $5 \mathrm{~h}$ after the start of a cycle were consolidated into a single temperature point by averaging $\sim 5,400$ recorded temperature data points in the 3 -h window containing the mostly linear portion of the melt. Using a set time after the start of the cycle is appropriate for this data because the timing of the freeze and melt are very consistent from cycle to cycle. The range over which the curves were averaged is shown as a blue bounding box in Figure 3. The start and end points of the range are somewhat arbitrary, and using different end points would affect the average temperature; however, the purpose here is to compare the curves, 


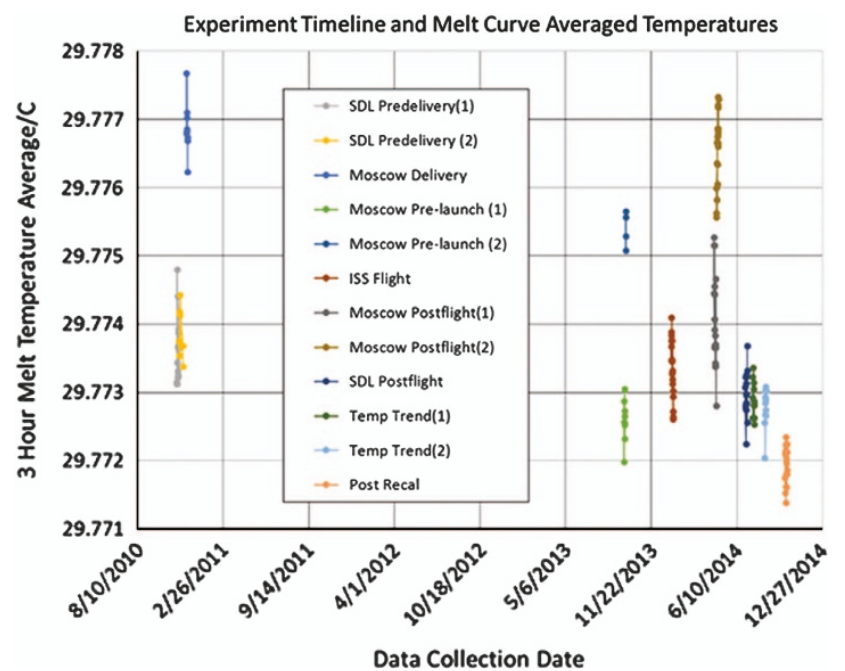

Figure 4. Melt curve averaged temperatures ( $2-5 \mathrm{~h}$ points) for the Ga-MOTR measurement sequences, from predelivery to a run taken after the final calibration and reassembly of the unit (see Table 1).

and the effect on the differences in melt average from changing the range is well below the variation seen from curve to curve.

To assure that any spikes in the data did not significantly affect the average, a sample of the individual curves was statistically analyzed and all the data were inspected. Most data points were consistent with a single Gaussian noise distribution with a s.d. of about $2.5 \mathrm{mK}$. A few noise spikes ( $<100$ points per 5,400 point curve) up to eight times the s.d. (randomly positive or negative) were present, but a worst case estimate of their effect on the average is only $0.030 \mathrm{mK}$.

Curve-averaged data for all measurement sequences from predelivery in 2010 to the completion of a post-calibration sequence in October 2014 are shown in Figure 4. Figure 5 shows the same average temperatures for each of the measurements in Figure 4, but also illustrates the trend in time for each of the measurement groups.

\section{DISCUSSION}

The data collected on ISS, at SDL, and the lower temperature Moscow post-flight group average at $29.7738^{\circ} \mathrm{C}$ which is $\sim 9 \mathrm{mK}$ higher than the published Ga melt temperature. This is consistent with offsets observed in bath testing and in cell thermal models and is owing to thermal gradients within the cell during melting of the PCM.

The lower temperature curve groups (Figure 4) cluster in the $29.773^{\circ} \mathrm{C}$ range, and three groups are out of family, $1-3 \mathrm{mK}$ higher. The root cause of this offset in these groups was not discovered, but it does not appear to be a drift in the thermistor over time since it occurs suddenly between adjacent runs or in the middle of a run. This temperature offset was observed between the two Moscow preflight runs and the two Moscow post-flight runs (Figure 4). In both cases, the second run was started within 1 day of the end of the first. This change is likely a systematic effect owing to environmental changes, which are not being recorded by the experiment hardware. In addition to the larger temperature variations discussed above, smaller drifts over time are seen in each measurement sequence (Figure 5). The data in Figure 4 and Figure 5 show that any drift in both the thermistor and the Ga melt point in this cell must be $<5 \mathrm{mK}$ peak to peak.

The average and s.d. of the melt temperature for ground data is $29.7738 \pm 0.0017^{\circ} \mathrm{C}$, for flight data is $29.7731 \pm 0.0008^{\circ} \mathrm{C}$, and for all data is $29.7737 \pm 0.0017^{\circ} \mathrm{C}$. The temperature accuracy is $11 \mathrm{mK}$,

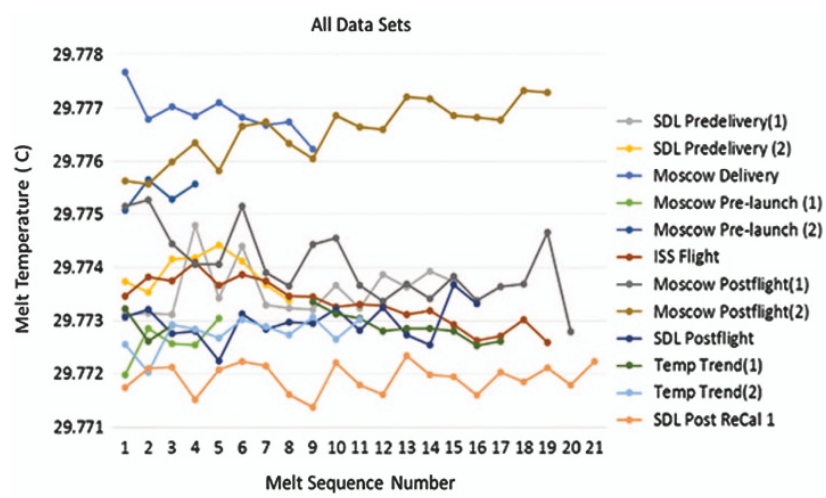

Figure 5. Melt curve averages for the data shown in Figure 4 plotted in the order collected for that series.

but the repeatability is considerably better. Averages for all the data groups are within $1 \mathrm{mK}$ of each other.

The primary objective of this effort was to determine if $\mathrm{Ga}$ melted at the same temperature in microgravity as it does on Earth. The data collected in Ga-MOTR in microgravity and on the Earth averaged within $0.6 \mathrm{mK}$ in this experiment. Within the precision of this data, this objective has been achieved. Concern for this issue arises from the extensive experiment base that exists on the value of microgravity for crystal growth studies. However, most of these studies center on the basic physics of crystal growth in large biological molecules and the development of crystals with minimum lattice disruptions.

Additional effort has been applied to the development of more perfect crystal structures for optical applications ${ }^{9}$ with a significant number of experiments devoted to the understanding of 'detached Bridgman growth processes. ${ }^{10}$ In this process, the crystals develop free from the wall of the container, and therefore do not include molecular scale wall structure disruptions. Separation of the PCM from the wall of the container in a microgram-thermal reference application, either by surface tension forces or detached Bridgman crystal growth, could lead to significant gradients and larger melt point errors than desired. The Ga could not detach from the walls of the cell used in this experiment because by design there was no air gap in the cell (the use of the bellows-shaped container in this experiment was to allow melt-freeze expansion while maintaining the PCM at near constant pressure without requiring an air gap). However, because $\mathrm{Ga}$ adhesion to stainless steel has been demonstrated in laboratory experiments, it is unlikely that Ga will exhibit the Bridgman effect in any stainless steel cell that does include an air gap. Laboratory experiments show droplets of liquid Ga adhering to stainless steel walls at forces greater than $1 \mathrm{~g}$ after incidental contact.

\section{CONCLUSIONS}

When the flight and ground data are compared, the difference in their ensemble means is $0.6 \mathrm{mK}$, with s.d. in the ground data of $1.7 \mathrm{mK}$. The ground data has a larger s.d. than the flight data because the larger ground data set includes more of the observed systematic variation. On the basis of these numbers, no significant difference between the melt temperature of $\mathrm{Ga}$ at $1 \mathrm{~g}$ and in microgram was observed.

As the ground data presented spans a period of collection of about four years, this experiment also demonstrates that $\mathrm{Ga}$ can be contained within a stainless steel container for at least 4 years without being contaminated by the container in a way significant enough to be detected by this hardware. This accuracy is well within the requirement for a remote-sensing thermal reference 
standard. Future work with Ga-based eutectics in a microgravity environment is planned by one of the authors. ${ }^{11}$

\section{ACKNOWLEDGMENTS}

Appreciation is expressed Mr Oleg Strugov, Russian Academy of Sciences, Institute of Biomedical Problems, Moscow, Russia for technical and flight of Ga-MOTR and to Cosmonaut Oleg Kotov (RSA) for conducting the experiment on the ISS

\section{CONTRIBUTIONS}

T.S.T. (guarantor) constructed the experiment hardware described here and performed most of the testing and data analysis. G.E.B. assisted with hardware design and data analysis and in getting the experiment funded and flown on the ISS. H.L. assisted with the testing, data processing, and analysis. I.P. performed Moscow ground testing of hardware and prepared necessary Russian support documents to gain approvals for space-flight experiments. V.S.S. is the IBMP team leader, coordinated the international effort, secured agreements with coordinating Russian organizations and the Russian Academy of Science and the Russian Space Agency elements to fly the experiment on ISS. A.B. performed initial ground investigations with gallium eutectics and advised SDL on procedures for preparing eutectics, realizing repeatable melts, and interpreting melt data.

\section{COMPETING INTERESTS}

The authors declare no conflict of interest.

\section{FUNDING}

This research was partially supported by NASA Contract NNX09AK44G.

\section{REFERENCES}

1 National Research Council. Earth Science and Applications from Space: National Imperatives for the Next Decade and Beyond. The National Academies Press: Washington, DC, 2007; 93-94.
2 Preston-Thomas H. The International Temperature Scale of 1990 (ITS 90). Metrologia 1990; 27: 3-10.

3 Mangum BW, Furukawa GT (eds). Guidelines for Realizing the International; Temperature Scale of 1990 (ITS-90). NIST Technical Note, 1990, 1265.

4 Burdakin A, Khlevnoy B, Samoylov M, Sapritsky V, Ogarev S, Panfilov A et al. Melting points of gallium and of binary eutectics with gallium realized in small cells. Metrologia 2008; 45: 2008.

5 Sapritsky V. Metrological support for climatic time series of satellite radiometric data. J Appl Remote Sens 2009; 3: 033506.

6 Burdakin A, Khlevnoy B, Samoylov M, Sapritsky V, Ogarev S, Panfilov A et al. Development of gallium and gallium-based small-size eutectic melting fixedpoints for calibration procedures on autonomous platforms. Int $J$ Thermophys 2008; 30: 20-35.

7 Yee JF, Lin M, Sarma K, Wilcox WR. The influence of gravity on crystal defect formation in InSb-GaSb alloys. J Cryst Growth 1975; 30: 185-192.

8 Su C, Sha Y, Lehoczky SL, Szofran FR, Gillies DC, Scripa RN et al. Crystal growth of $\mathrm{HgZnTe}$ alloy by directional solidification in low gravity environment. J Cryst Growth 2002; 234: 487-497.

9 Sakata K, Mukai M, Rajesh G, Mukannan A, Inatomi Y, Ishikawa T et al. Thermal properties of molten InSb, GaSb, and InxGa1-xSb alloy semiconductor materials in preparation for crystal growth experiments on the International Space Station. Adv Space Res 2013; 53: 689-695.

10 Croll A, Volz MP. Detached Bridgman growth-a standard crystal growth method with a new twist. MRS Bull 2009; 34: 245-250.

11 Burdakin A, Khlevnoy B, Krutikov V, Panfilov A, Puzanov A, Samoylov M et al. Upcoming space experiments for developing space-borne low-temperature fixedpoint blackbody. Proc NEWRAD 2014; 2014: 57-58.

(c) (\$) $\Theta$ This work is licensed under a Creative Commons Attributionc. NonCommercial-NoDerivatives 4.0 International License. The images or other third party material in this article are included in the article's Creative Commons license, unless indicated otherwise in the credit line; if the material is not included under the Creative Commons license, users will need to obtain permission from the license holder to reproduce the material. To view a copy of this license, visit http:// creativecommons.org/licenses/by-nc-nd/4.0/ 